\title{
Conhecimento Matemático Para O Ensino De Escala Apresentada Em Gráficos Nos Anos Iniciais Do Ensino Fundamental
}

\author{
Mathematical Knowledge For The Teaching Of Scale Presented In Graphs In The \\ Primary School
}

\author{
Milka Cavalcanti* \\ Universidade Federal de Pernambuco - UFPE \\ Gilda Guimarães** \\ Universidade Federal de Pernambuco - UFPE
}

\begin{abstract}
Resumo
Este estudo buscou estabelecer Conhecimentos Matemáticos para o Ensino de escala (Mathematical Knowledge for Teaching - MKT, Ball e colaboradores 2008) apresentada em gráficos estatísticos. A partir da revisão da literatura e de uma pesquisa diagnóstica com crianças e adultos dos anos iniciais, estabelecemos as habilidades necessárias para a compreensão de escala nos anos iniciais para cada tipo de Conhecimento Matemático para o Ensino. O Conhecimento Matemático para o Ensino de Escala não deve ser apenas teórico/epistemológico, mas deve instrumentalizar o professor para reconhecer uma resposta errada, dimensionar a natureza do erro, ter familiaridade com os erros comuns cometidos pelos alunos, planejar uma abordagem de ensino capaz de superar o erro, conhecer o livro didático e o currículo e também compreender que tipo de complexificação pode ser feita ao trabalhar este conceito no evoluir da escolaridade.
\end{abstract}

Palavras-chave: Estatística, Escala, Anos Iniciais, Conhecimentos dos Professores.

\begin{abstract}
This study aimed to establish Mathematical Knowledge for Scale Teaching (Ball et al, 2008) presented in statistical graphs. From the review of the literature and a diagnostic survey with children and adults of primary school, we established the necessary skills for the comprehension of for each type of Mathematical Knowledge for Teaching. Mathematical Knowledge for Scale Teaching should not be only theoretical/epistemological, but should allow the teacher to recognize a wrong answer, the nature of the mistake, be familiar with the common mistakes made by the students, plan an approach to teaching that can overcome the error, know the textbook and curriculum and also understand that type of complexity can be made by working with this concept in the evolution of schooling.
\end{abstract}

Keywords: Statistic, Scale, Primary school, Teacher Knowledge.

\footnotetext{
* Doutora em Educação Matemática e Tecnológica- UFPE. Professora da rede pública e privada de Recife/PE. mirgca@gmail.com

${ }^{* *}$ Doutora em Psicologia Cognitiva - UFPE. Professora da Pós Graduação em Educação Matemática e Tecnológica - UFPE, gilda.lguimaraes@gmail.com
} 


\section{Ensino De Estatística - Escala}

No mundo contemporâneo temos observado que as representações gráficas têm sido utilizadas com uma grande frequência como um importante instrumento para a organização de ideias de diferentes áreas e sobre variados temas. O principal argumento utilizado para justificar o uso destas representações é que elas facilitam a transmissão de informações por apresentarem de forma resumida e rápida um quantitativo amplo de dados.

Se desejamos alunos capazes de compreender o mundo físico e social, é preciso que eles sejam letrados estatisticamente, que segundo Gal (2002), é definido como a capacidade de interpretar, avaliar criticamente e comunicar informações e mensagens estatísticas. Sendo assim, ter acesso às informações estatísticas não é suficiente. É fundamental que as pessoas desenvolvam a capacidade de interpretar criticamente os dados, elaborar gráficos que sistematizem informações, além de comunicar e/ou discutir suas ideias a partir deles.

Esta relevância social fez com que o trabalho com Estatística fosse incluído no currículo brasileiro (PCN's, 1997 e BNCC, 2018) desde os anos iniciais do Ensino Fundamental. Nesses documentos a Estatística é um dos eixos da área de Matemática, mas que não se restringe à mesma, uma vez que possibilita articular as ideias matemáticas através de um trabalho conjunto com outras áreas do saber. Nos anos iniciais, essa articulação é potencializada uma vez que seu ensino é realizado pelo professor polivalente, ou seja, aquele que ensina todas as disciplinas.

Estas informações estatísticas geralmente são apresentadas em representações gráficas e ao observá-las é possível constatar que os eixos e a escala são elementos extremamente importantes para a estrutura do gráfico. São elas que irão fornecer as informações quantitativas sobre os dados que estão sendo apresentados e influenciam diretamente no aspecto visual dos dados apresentados.

Alguns estudos investigaram a compreensão de crianças sobre escalas apresentadas em gráficos (Tierney e Nemirovsky, 1991; Tierney, Weinberg e Nemirovsky, 1992; Ainley, 2000; Guimarães, Gitirana e Roazzi, 2001; Lima e Magina, 2004; Cavalcanti e Guimarães, 2016, entre outros). Esses estudos evidenciam que as crianças apresentam compreensões parciais sobre a função da escala e que têm dificuldades em compreender as unidades e subunidades dos intervalos da reta numérica apresentada na escala do gráfico. Nessa mesma linha outros estudos (Cavalcanti, 2010; Lima, 2010; INAF, 2016) evidenciam que adultos com baixa escolarização também apresentam dificuldades em compreender a escala apresentada em 
um gráfico de barras.

Guimarães (2016) argumenta que o cotidiano, a maturidade cognitiva e a escolarização, de modo geral, não são suficientes para o desenvolvimento mais avançado em relação à compreensão da escala apresentada em gráficos. Por isso, a autora defende que o papel da escola é de propiciar aos alunos contato com variadas situações significativas para a aprendizagem dos mesmos.

O estudo de Evangelista (2014) vem ratificar o papel da escola, visto que quando alunos de $5^{\mathrm{a}}$ ano foram levados a refletir sobre escala representada em gráficos de barras e de linhas em uma intervenção de ensino, com duração de 2 (dois) dias de aulas de aproximadamente 2 horas, eles demonstraram capacidade e facilidade para aprender.

Entretanto, Cavalcanti, Natrielli e Guimarães (2010) levantaram que o uso que é feito das representações gráficas na mídia impressa muitas vezes compromete as informações. As autoras investigaram os gráficos que eram apresentados em três suportes de informações (jornal e revistas) e perceberam que 39\% deles apresentavam erro em relação à escala dos gráfícos. As autoras afirmam também que consideram esse resultado muito importante, pois com a grande tecnologia disponível aos profissionais de arte gráfica, esses erros podem ser considerados intencionais, com o intuito de manipular os dados.

Partindo destas inquietações sobre o uso inadequado das escalas pela mídia impressa, o baixo desempenho de alunos, tanto adultos como crianças, na compreensão da escala dos gráficos e a facilidade que as crianças apresentaram em aprender quando levadas a isso, acreditamos que é fundamental que a escola proponha um trabalho sistematizado com representações gráficas.

Para tal é necessário considerar os diferentes tipos de gráficos e as diferentes unidades escalares. Esse trabalho deve ser aliado também à compreensão de diferentes grandezas, principalmente a grandeza comprimento, discutindo as unidades de medidas e suas subunidades, para que de fato possamos ter cidadãos capazes de serem críticos diante da realidade física ou social.

Dessa forma, para que a escola possa desempenhar seu papel de formadora de cidadãos faz-se necessários que os professores sejam capazes de realizar processos de ensino aprendizagem significativos para os alunos. Entretanto, diversos estudos (Shaughnessy, Garfield e Greer, 1996; Monteiro e Selva, 2001; Lima, Silva, Rodrigues e Feitosa, 2006; Silva, Amaral, Albuquerque e Oliveira, 2006; Lemos e Gitirana, 2007; Rossini, 2006; Arteaga, Batanero e Ruiz, 2008; Artega, Batanero e Cañadas, 2012) têm mostrado que tanto em nível 
internacional como nacional os professores dos anos iniciais não estão preparados para o trabalho com as representações gráficas e, em específico, com a escala apresentada em gráficos.

Dessa forma, é cada vez mais urgente a necessidade de pensarmos um processo de formação de professores dos anos iniciais do Ensino Fundamental que seja capaz de trabalhar de forma significativa o ensino de Estatística com o intuito possibilitar uma formação que interfira diretamente no processo de ensino aprendizagem dos alunos.

Para refletirmos sobre os saberes necessários para ensinar nos apoiamos em Ball, Thames e Phelps (2008), que nos apresentam seis tipos de conhecimentos matemáticos necessários para o ensino: Conhecimento Comum do Conteúdo, Conhecimento Especializado do Conteúdo, Conhecimento do Horizonte do Conteúdo, Conhecimento do Conteúdo e do Aluno, Conhecimento do Conteúdo e Ensino, Conhecimento do Conteúdo e Currículo.

Acreditamos que para que os professores sejam capazes de superar suas lacunas de formação em relação à escala apresentada em gráficos e possam levar seus alunos a também superar as dificuldades, não é suficiente o conhecimento matemático do conteúdo a ser ensinado. Consideramos, assim como Ball et al (2008), que existe uma multiplicidade de saberes que os docentes precisam mobilizar para o ensino de um conteúdo. O conhecimento dos professores precisa ir além de identificar se o aluno apresentou uma resposta certa ou errada para uma determinada situação, mas envolve outras habilidades relacionadas a reconhecer a natureza dos erros, ser capaz de identificar as estratégias utilizadas pelos alunos e propor atividades capazes de superá-las.

\section{Conhecimento Matemático Para O Ensino - MKT}

Ball, Thames e Phelps (2008) desenvolveram a noção de Mathematical Knowledge for Teaching (MKT) - Conhecimento Matemático para o Ensino. (Figura 1).

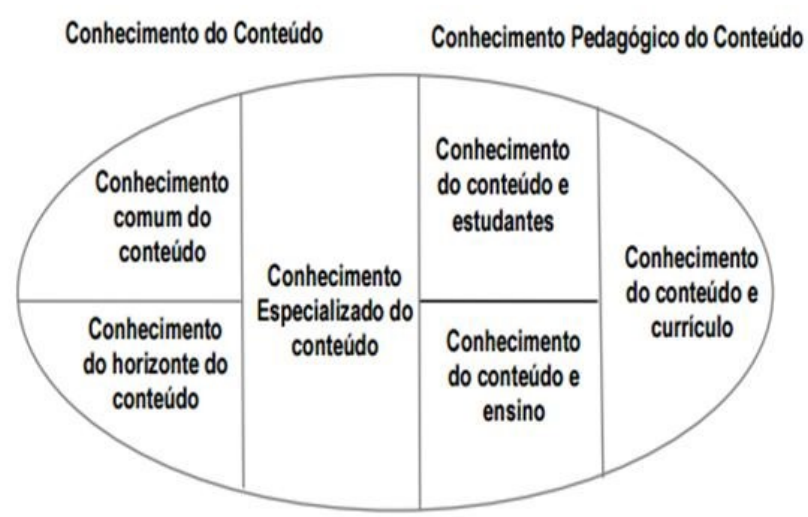

Figura 1 - Diagrama dos domínios do Conhecimento Matemático para o Ensino Fonte: BALL; THAMES; PHELPS, 2008, p. 404 
O "Conhecimento Comum do Conteúdo" diz respeito ao conhecimento que é utilizado em uma variedade de situações e não apenas para o ensino. Pode ser considerado como a capacidade de identificar se uma resposta está errada ou não, efetuar um cálculo ou resolver um problema matemático corretamente e até mesmo ser capaz de identificar quando um livro didático é impreciso ou possui definições inadequadas.

O segundo tipo, o "Conhecimento Especializado do Conteúdo", está ligado diretamente ao ensino e relaciona-se à capacidade de reconhecer e analisar a natureza e os padrões de erros que os alunos geralmente cometem ao resolver uma determinada situação. Além disso, envolve também escolher e explorar definições adequadamente, utilizando diferentes representações e ser capaz de explicar regras e procedimentos.

Já o "Conhecimento do Conteúdo e do Aluno" combina o conhecimento sobre o conteúdo matemático e dos alunos correspondendo à capacidade do professor ter familiaridade com os diversos erros e dificuldades dos alunos. Existem poucos estudos que investigam o que os professores sabem avaliar acerca do conhecimento e das estratégias dos alunos. Desta forma é um conhecimento que engloba questões cognitivas, especificamente sobre como os alunos aprendem e pensam sobre a Matemática.

Eles definem o "Conhecimento do Conteúdo e do Ensino" como sendo a capacidade de identificar abordagens e intervenções de ensino para superar os erros dos alunos ou que sejam capazes de explorar certos aspectos relevantes a compreensão de um conteúdo. Implica em ter clareza sobre os objetivos da aula, avaliar quais exemplos devem ser utilizados para introduzir um conteúdo, identificar qual atividade é mais fácil ou mais adequada para um aluno, quando devem propor uma nova questão ou tarefa. Deste modo, consideramos que ele caminha muito além de apenas conhecer o conceito a ser ensinado.

Além desses, os autores também citam a importância do "Conhecimento do Horizonte do Conteúdo" que compreende como um conteúdo deve ser abordado em cada ano de ensino e como ele vai se complexificando. Finalmente, o "Conhecimento do Conteúdo e do Currículo" que se relaciona a conhecer materiais, livros didáticos, currículos e orientações metodológicas acerca de um conteúdo matemático.

Ball e Bass (2009) acreditam que a investigação sobre o Conhecimento do Horizonte do Conteúdo merece uma atenção especial, na medida em que esse conhecimento parece orientar os professores na tomada de decisões que podem contribuir fortemente para o desenvolvimento do pensamento matemático dos seus alunos, permitindo a exploração eficiente e proveitosa de um imenso panorama matemático, indo além das fronteiras estabelecidas pelos 
programas curriculares.

Deste modo, para ser um professor não é suficiente saber o conteúdo a ser ensinado, indo muito além e envolvendo a habilidade de ter um arcabouço de habilidades que sejam capazes de ajudar os seus alunos a superar suas dificuldades.

Neste artigo temos como objetivo estabelecer relação entre os estudos anteriores que abordaram a escala apresentada em gráficos e a teoria do Mathematical Knowledge for Teaching - MKT para definir os diferentes tipos de Conhecimentos Matemáticos para o Ensino de escala apresentada em gráficos estatísticos. Nossa preocupação com as escalas apresentadas nos gráficos estatísticos é com o desenvolvimento da possibilidade de elaborar, compreender e questionar essas representações, identificando intenções e possíveis manipulações de quem a produz.

Consideramos que este estudo será capaz de fornecer subsídios para nortear o processo de formação docente, visto que as pesquisas têm apontado para a existência de grandes lacunas na formação inicial e continuada dos professores mundialmente.

\section{Metodologia}

Para alcançar o objetivo de estabelecer relação entre os estudos anteriores que abordaram a escala apresentada em gráficos e a teoria do Mathematical Knowledge for Teaching - MKT partimos de um grande e exaustivo levantamento de estudos teóricos na literatura nacional e internacional a fim de extrair deles os elementos constituintes dos conhecimentos necessários para o ensino de escala. Isto foi necessário, pois a teoria de Ball e colaboradores apresenta quais são os seis tipos de Conhecimentos Matemáticos para o Ensino e o que nos inquietava era estabelecê-los para o conceito de escala apresentadas em gráfícos. Esses estudos estão apresentados na revisão da literatura apresentados no item 1 desse artigo e em cada tipo de conhecimento que será discutido a seguir.

\section{Resultados}

Inicialmente sentimos uma grande dificuldade em conseguir estabelecer e definir diferenças e conexões entre cada um dos conhecimentos apresentados na Teoria de Ball e colaboradores a partir do conceito de escala. Em seguida, nos deparamos com um resumido quantitativo de estudos que investigavam sobre a escala apresentada em gráficos. Alguns envolviam apenas alunos, nos variados anos ou módulos dos anos iniciais do Ensino 
Fundamental, uns com a habilidade de construção, outros com a de interpretação ou as duas. Alguns só pontuavam se acertavam ou erravam e outros analisavam as estratégias. Em relação aos professores também a situação era parecida e, muitas vezes, as análises não se detinham minuciosamente nas habilidades e diferentes tipos de conhecimentos envolvidos. Tampouco encontramos estudos que abarcassem quais habilidades precisam ser compreendidas para a interpretação e construção de gráficos, considerando uma complexificação, nessa faixa de escolarização.

\section{Conhecimento Comum de Escala}

Esse tipo de conhecimento matemático envolve compreender o conceito de escala apresentada em gráfico, mas não é exclusivo do professor que ensina Matemática:

a) Conhecimento Comum de Escala para interpretar uma escala refere-se à capacidade do professor localizar corretamente um valor na escala.

b) Conhecimento Comum de Escala para construir uma escala refere-se à capacidade do professor construir corretamente a escala do gráfico, considerando a proporcionalidade dos intervalos.

Em relação à interpretação sabemos que é imprescindível que o indivíduo compreenda a noção de eixo de coordenadas para que seja capaz de localizar os valores da escala.

Friel, Curcio e Brigh (2001) afirmam que muitas vezes as pessoas são capazes de desenhar ou ler uma determinada informação na escala, mas têm pouca ideia de como escolher uma escala adequada para um determinado conjunto de dados a serem representados no gráfíco.

Desta forma, a construção é uma habilidade mais complexa, pois envolve uma série de etapas que irão determinar o sucesso na atividade. De acordo com Vanin (2009), na construção de um gráfico a primeira tarefa importante que temos que realizar é uma escolha conveniente do intervalo da escala e para isso precisamos analisar o tamanho do papel, identificar os valores máximos e mínimos das grandezas que serão representadas e, a partir dessas dimensões, calcular a escala que permita ocupar o espaço disponível. Em seguida precisamos fazer a divisão da escala de modo a localizar e marcar os pontos facilmente, bem como permitir uma leitura posterior de valores a partir do gráfico, mas é imprescindível que os espaços entre os intervalos das escalas sejam proporcionais.

\section{Conhecimento Especializado de Escala}

É um tipo de conhecimento que está relacionado ao ensino, portanto é um conhecimento específico dos professores. Esse se refere à capacidade do docente em analisar e 
adequar os conceitos às necessidades dos alunos, bem como reconhecer padrões de erros dos mesmos ao lidarem com determinadas atividades; responder seus "porquês", entre outros aspectos:

a) Conhecimento Especializado de Escala para interpretar: Compreender que um gráfico pode apresentar diferentes intervalos escalares (unitário; 2 em 2; 10 em 10, etc.); Compreender que existe uma dificuldade diferente entre os alunos localizarem em uma escala valores explícitos e implícitos.

b) Conhecimento Especializado de Escala para construir: Compreender que os intervalos devem ser proporcionais; Compreender que os diferentes conjuntos numéricos (números naturais, racionais, inteiros...) e a grandeza dos números irão influenciar no intervalo da escala.

$\checkmark$ Explorar definições adequadamente, fazendo uso dos termos estatísticos adequados a uma escala (unidade, subunidade, intervalo, valor explícito, valor implícito, proporcional...);

$\checkmark$ Saber explicar regras e procedimentos para a construção e a interpretação de uma escala.

Alguns estudos diagnósticos como os desenvolvidos por Guimarães, Gitirana e Roazzi (2001) e Cavalcanti e Guimarães (2016) já chamavam nossa atenção para alguns aspectos relacionados à escala das representações gráficas por dificultar ou facilitar o processo de interpretação ou construção da mesma. Guimarães et al (2001) afirmam que nas questões nas quais os valores eram apresentados na escala, o que exigia que inferissem um valor no intervalo da reta numérica (escala), o percentual de acertos foi muito baixo do que nas situações em que os valores estavam explícitos na escala. Esses autores levantam assim, que a localização de um valor implícito na escala é um marcador de dificuldade para os alunos. Em relação à habilidade de construção, o mesmo estudo aponta que quando não é possível estabelecer uma relação uma a um na escala, gerando a necessidade de uma escala diferente de 1 , a dificuldade foi muito maior. Aí vemos a importância de se considerar os valores que serão representados, visto que o conjunto numérico e a grandeza dos números também irão implicar em dificuldades distintas.

Cavalcanti e Guimarães (2016) investigaram quatro variáveis para a compreensão da escala apresentada nos gráficos: o tipo de gráfico; o valor da escala; a necessidade de o aluno localizar um valor implícito ou explícito na escala, ou de localizar uma frequência ou uma categoria a partir da escala. A partir deste estudo também elencamos mais um aspecto que é o valor da escala, visto que nos resultados as autoras pontuam que a localização de valores em escala unitária foi mais fácil para os alunos investigados. Logo, é imprescindível também levar em consideração os diferentes intervalos escalares (1 em 1, 2 em 2, 10 em 10, etc.) 
E por fim, a importância da necessidade de estabelecer a proporcionalidade entre os pontos a serem representados no momento de construir uma representação gráfica. Do contrário o que temos é uma representação que não apresenta de forma fidedigna os dados, implicando em erro ou em manipulação de informações.

\section{Conhecimento do Horizonte de Escala}

Esse conhecimento está relacionado à capacidade do professor em compreender como vai se complexificando a compreensão do conceito, ou seja, como se dá a gradação das aprendizagens. O professor precisa desse conhecimento para relacionar o que os alunos sabem com o que ele tem como objetivo que os alunos aprendam naquela aula, no semestre ou no ano escolar, considerando o desenvolvimento do conceito em questão para aquele nível de ensino. Os estudos da psicologia cognitiva, em geral, são base para a compreensão dessa gradação. Tal conhecimento orienta os professores na tomada de decisões para o desenvolvimento da aprendizagem dos alunos indo além das fronteiras estabelecidas pelos programas curriculares e outros documentos oficiais.

Não encontramos estudos que apresentassem uma progressão para o ensino de escala nos anos iniciais do Ensino Fundamental, apenas estudos com diferentes faixas etárias com diferentes instrumentos. Porém percebemos que as pesquisas vêm trazendo indícios que é possível, mas não existe nenhuma sistematização disso.

Selva (2003) evidenciou que crianças de 5-6 anos de idade foram capazes de resolver atividades utilizando barras de blocos (LEGO) recobertas com papel. Guimarães (2010) também trabalhando com crianças de 5 anos de idade observou que as mesmas foram capazes de preencher e compreender a construção de um gráfico de barras em papel quadriculado em situação de jogo.

Desta forma, aspectos fundamentais para determinar a evolução e complexidade do trabalho com escala não foram possíveis de ser identificados nos estudos levantados, uma vez que não encontramos pesquisas que avaliassem com todos os anos, todas as habilidades para o trabalho com escala, além de que partiam sempre de instrumentos de coleta distintos. Conforme Error! Reference source not found.1.

Quadro 1 - Estudos envolvendo interpretação ou construção de gráficos e turmas investigadas

\begin{tabular}{|l|l|l|}
\hline Estudo & Turma & Habilidade \\
\hline $\begin{array}{l}\text { Tierney e Nemirovsky } \\
(1991)\end{array}$ & $5^{\circ}$ ano & Construção \\
\hline
\end{tabular}




\begin{tabular}{|l|l|l|}
\hline $\begin{array}{l}\text { Tierney, Weinberg e } \\
\text { Nemirovsky (1992) }\end{array}$ & $5^{\circ}$ ano & Construção \\
\hline Ainley (2000) & Crianças de 6 anos & Construção \\
\hline $\begin{array}{l}\text { Guimarães, Gitirana e } \\
\text { Roazzi (2001) }\end{array}$ & $4^{\circ}$ ano & Interpretação e Construção \\
\hline Lima e Magina (2004) & $5^{\circ}$ ano & Interpretação e Construção \\
\hline Cavalcanti (2010) & $\begin{array}{l}3^{\circ} \text { e 50 anos e Módulos I- } \\
\text { II e III da Eja }\end{array}$ & Interpretação \\
\hline Lima (2010) & $\begin{array}{l}\text { Anos iniciais, Anos Finais } \\
\text { e Ensino Médio da Eja }\end{array}$ & Interpretação e Construção \\
\hline $\begin{array}{l}\text { Patrocínio e Guimarães } \\
(2007)\end{array}$ & Módulo II da Eja & Interpretação e Construção \\
\hline Selva (2003) & Crianças de 6 anos & Interpretação e Construção \\
\hline Evangelista (2014) & $5^{\circ}$ ano & Interpretação e Construção \\
\hline
\end{tabular}

Fonte: as autoras

Uma vez que não foi possível, a partir da literatura, definir os seis tipos de conhecimentos apresentados por Ball et al (2008) realizamos uma pesquisa com a finalidade de traçar o Conhecimento do Horizonte de Escala. Preocupamo-nos em apresentar uma complexificação o mais próxima da realidade do sistema educacional no qual estamos inseridos. Não queríamos apresentar como resultado uma progressão para o ensino de escala desenvolvido no interior da academia a partir de inferências da realidade. Como a própria teoria aponta, os estudos da psicologia cognitiva, em geral, são base para a compreensão dessa gradação.

Para isso, elaboramos um teste considerando as seguintes variáveis levantadas pelos estudos anteriores da área: reconhecimento de elementos estruturais (título, eixos e fonte), localização de valores explícitos e implícitos na escala de diferentes tipos de gráfico e com diferentes intervalos escalares, além da construção de gráficos com conjuntos numéricos com valores baixos e altos. Esse teste foi respondido por 210 alunos do $1^{\circ}$ ao $5^{\circ}$ ano do Ensino Fundamental (crianças e adultos da EJA).

A partir do desempenho dos alunos elaboramos a proposta apresentada no Quadro 1. Ele expõe nossas conclusões acerca de como pode ocorrer a progressão no ensino de escala apresentada em gráficos. O primeiro aspecto que é possível verificar é que consideramos que esse trabalho deve acontecer em todos os anos e módulos. 
Quadro 1 - Progressão para o ensino de escala

\begin{tabular}{|c|c|c|c|c|c|c|c|}
\hline Habilidades por ano & $1^{0}$ & $2^{0}$ & $\mathbf{3}^{\circ}$ & $4^{0}$ & $5^{0}$ & $\begin{array}{l}\text { Mod. } \\
\text { I e } \| \\
\text { EJA }\end{array}$ & $\begin{array}{l}\text { Mod. } \\
\text { III } \\
\text { EJA }\end{array}$ \\
\hline \multicolumn{8}{|l|}{$\begin{array}{l}\text { Reconhecimento de elementos estruturais (título, } \\
\text { eixos e fonte) }\end{array}$} \\
\hline \multicolumn{8}{|l|}{$\begin{array}{l}\text { Localização de valores explícitos em gráficos de } \\
\text { barras com escala unitária e pictogramas }\end{array}$} \\
\hline \multicolumn{8}{|l|}{$\begin{array}{l}\text { Construção de pictogramas e gráficos de barras } \\
\text { com escala unitária. }\end{array}$} \\
\hline \multicolumn{8}{|l|}{$\begin{array}{l}\text { Localização de valores explícitos do gráfico de } \\
\text { barras com escala nâo unitária; }\end{array}$} \\
\hline \multicolumn{8}{|l|}{$\begin{array}{l}\text { Construção: Pictogramas e gráficos de barras } \\
\text { com escala năo unitária. }\end{array}$} \\
\hline \multicolumn{8}{|l|}{$\begin{array}{l}\text { Localizaçâo de valores implícitos em escala } \\
\text { unitária ou nấo em gráfico barras ou linha; }\end{array}$} \\
\hline \multicolumn{8}{|l|}{$\begin{array}{l}\text { Identificação de erros de proporcionalidade em } \\
\text { gráficos; }\end{array}$} \\
\hline \multicolumn{8}{|l|}{$\begin{array}{l}\text { Identificação do impacto causado na informação } \\
\text { se mudarmos o intervalo da escala do gráfico. }\end{array}$} \\
\hline $\begin{array}{l}\text { Adequação do tipo de gráfico em função dos } \\
\text { dados a serem representados. }\end{array}$ & & & & & & & \\
\hline
\end{tabular}

Fonte: as autoras

Em relação às habilidades, a primeira: "Reconhecimento de elementos estruturais (título, eixos e fonte)", consideramos importante em todos os anos/módulos, pois é a partir desta análise que se compreende que tipo de informação está sendo apresentada no gráfico. Em seguida realizamos uma gradação envolvendo duas principais habilidades: a de localização e de construção. Quanto à de localização o que varia entre elas é se deve localizar um valor explícito ou implícito e também se o intervalo da escala é unitário ou não. Já a de construção nossa preocupação foi se o aluno teria que construir uma escala unitária ou não. As demais habilidades envolvem uma análise mais acurada da representação a fim de identificar se existe manipulação das informações, por isso acreditamos que devem ser abordadas nos anos/módulos mais avançados.

Julgamos, que nossa proposta de progressão para o ensino deste conteúdo (Conhecimento do Horizonte de Escala), não deve ser uma sugestão engessada. A maior contribuição que ela trará para o ensino é deixar claro para os professores que variáveis (habilidade de construção ou interpretação; intervalo escalar; localização de valor implícito ou explícito; grandeza do conjunto numérico) precisam ser consideradas ao se trabalhar com o conceito de escala e que tipo de variações podem ser realizadas para que se aprofunde ou simplifique a abordagem destas variáveis. 
É importante relembrar que percebemos que os alunos demonstraram ser capazes de aprender sobre este conteúdo, entretanto, o currículo brasileiro não propõe o seu ensino para os anos iniciais no eixo de Estatística. Logo, esta proposta de progressão também pode suprir esta lacuna e ser vista como um norte para se pensar o ensino em cada ano ou modalidade dos anos iniciais do Ensino Fundamental.

Conhecimento de Escala e do Aluno

É a combinação do saber do aluno e da Matemática, no caso o conceito de escala. É o professor ser capaz de compreender as dificuldades e facilidades dos alunos e ter familiaridade com as estratégias utilizadas para interpretar ou construir a escala dos gráficos.

a) Conhecimento de Escala e Aluno para Interpretar - Ser capaz de identificar as seguintes dificuldades nos alunos ao interpretar a escala:

- Compreender a relação entre os eixos de coordenada de um gráfico.

- Compreender os valores intermediários ou subunidades da escala.

b) Conhecimento de Escala e Aluno para Construir - Ser capaz de identificar as seguintes estratégias dos alunos ao apresentarem dificuldades com a construção da escala:

- Atribuir à origem da escala o menor valor a ser registrado ou, no gráfico pictórico, desconsiderar uma linha de base.

- Utilizar ícones que não têm o mesmo tamanho ou espaçamento.

- Colocar os valores em cima das barras ou linhas, mas não atendendo a proporcionalidade.

- Registrar na escala os valores sem considerar a ordem crescente expressa em uma reta numérica.

- Colocar os valores em ordem crescente, mas sem proporcionalidade entre os intervalos.

Quanto à interpretação nos baseamos no estudo de Cavalcanti e Guimarães (2016) que apontam como estratégias utilizadas pelos alunos ao interpretarem os gráficos, devido a não compreensão da escala e por não conseguirem localizar valores implícitos, dar como respostas valores aproximados acima ou abaixo. Esta estratégia demonstra uma dificuldade em relação à interpretação da escala que é não compreender que ela é formada também por valores intermediários ou subunidades que precisam ser consideradas. Tierney e Nemirovsky (1991) afirmam que os alunos, frequentemente, não mantinham um espaço constante entre os valores marcados na escala, evidenciando a incompreensão com a reta numérica. 
Ainley (2000) observou que crianças de 6 anos conseguiam construir gráficos, mas não colocavam os eixos, o título e a escala.

Evangelista (2014) também apontam algumas estratégias como fazer uma lista com as informações da tabela a ser representada; colocar os nomes ou valores em cima das barras, mas sem proporcionalidade; fazer barras proporcionais, com ou sem escala.

Estes estudos apontam estratégias comuns que são utilizadas por alunos que ainda apresentam dificuldades com a construção de uma escala e que podem ser entendidas como um momento em que o aluno está formulando hipóteses sobre um novo conceito.

\section{Conhecimento de Escala do Ensino}

É um tipo de conhecimento que visa à relação entre o ensino e a Matemática (escala). Envolve o planejamento do ensino, a preparação de atividades, a avaliação e estratégias para superar as dificuldades dos alunos. Para tal, precisam ser consideradas também as crenças dos professores sobre a Matemática, suas concepções de ensino e aprendizagem, suas visões sobre os alunos e o contexto da sala de aula.

Nós consideramos que ao planejar uma aula para introduzir o conceito de escala apresentada em gráficos ou para superar as dificuldades dos alunos é importante considerar os seguintes aspectos:

- Explorar o trabalho com as habilidades representar, localizar, analisar, comparar e construir escalas em diversas situações e com diferentes unidades escalares durante as aulas.

- Variar as situações de ensino e uso do conceito de escala - atividades de medida de comprimento, de reta numérica e de mapas.

Baseamo-nos em diversos estudos, como o de Selva (2003), que nos evidenciam que é possível trabalhar com escala já nos anos iniciais, desde que adotemos metodologias adequadas a cada faixa etária.

Lima e Magina (2004) apontam que a construção de gráficos utilizando diferentes escalas parece ter favorecido a compreensão de conhecimentos fundamentais à leitura e interpretação de gráficos, tais como a ideia de proporcionalidade existente no eixo vertical, expressa pela distância entre dois pontos, associada à ideia de sequência numérica para estimar o valor que não estava explicitamente apresentado no eixo.

Patrocínio e Guimarães (2007) desenvolveram aulas trabalhando diferentes habilidades: interpretação e construção de gráficos de barras e perceberam a possibilidade de 
aprendizagem deste conceito.

Evangelista (2014) desenvolveu sessões de intervenções de ensino do conceito de escala de forma intencional e sistemática, explorando diferentes habilidades: representar, localizar, analisar, comparar e construir escalas em diversas situações e com diferentes unidades escalares, possibilitando aos alunos reflexões sobre o tema em questão. O processo de intervenção variava também as situações de uso do conceito de escala - medida de comprimento, reta numérica e mapas.

Esses estudos ora envolvem crianças ora adultos com pouca escolarização e ambos os grupos são beneficiados com breves processos de intervenção.

\section{Conhecimento de Escala e do Currículo}

É um tipo de conhecimento que envolve conhecimentos sobre os programas curriculares estabelecidos, os materiais didáticos, os livros didáticos e recursos metodológicos para um determinado nível de ensino e suas finalidades. Consideramos como Conhecimento de Escala e Currículo o professor ser capaz de:

- Conhecer o que os programas curriculares oficiais (atualmente a BNCC) prescrevem para o trabalho com escala.

- Conhecer o que os livros didáticos propõem para o trabalho com escala.

No caso específico de escala, Cavalcanti e Guimarães (2016) em estudo anterior, verificaram que tanto os PCN's referentes aos anos iniciais do Ensino Fundamental como a Proposta Curricular para a Educação de Jovens e Adultos referente aos anos iniciais do Ensino Fundamental ( $1^{\circ}$ Segmento) fazem referência ao trabalho com escala apenas nos eixos de Números e Operações e Grandezas e Medidas. Nos demais eixos e em específico no eixo de Tratamento da Informação o texto da proposta curricular não faz menção a esse elemento gráfico.

Apenas no Guia do PNLD 2016 é afirmado que é desejável que seja mais bem desenvolvida a construção dos vários tipos de gráficos, com valorização de seus diferentes elementos, como título, escalas, legendas, títulos e variáveis dos eixos, proporcionalidade nas representações dos dados (nos casos das escalas lineares, mais adequadas a essa fase de escolaridade).

Quanto ao livro didático, estudos (Pagan, Leite, Magina e Cazorla, 2008; Guimarães, Gitirana e Roazzi, 2001; Evangelista e Guimarães, 2013; Díaz, Batanero, Arteaga e Serrano, 2015), em nível nacional e internacional vêm mostrando que o foco das atividades com gráficos é nas habilidades de interpretação, deixando de lado a construção. O tipo de gráfico mais 
encontrado é o de barras. Quanto à escala ela é pouco explorada.

Concordamos que as pesquisas desenvolvidas e as reflexões levantadas por Ball e sua equipe trazem contribuições relevantes para a discussão sobre a formação do professor que ensina Matemática. Em particular, entendemos que a defesa da existência de um rol de conhecimentos necessários para que o professor possa educar matematicamente seus alunos é pertinente, pois leva em consideração toda a complexidade do processo de ensino aprendizagem.

O Quadro 3 foi criado após o estabelecimento nas relações apresentadas anteriormente para cada conhecimento e também a partir de vários momentos de discussões e análise dos Conhecimentos Docentes para o Ensino de Escala. Nele fica claro quais são as habilidades que consideramos importantes que os professores mobilizem no momento de desenvolver o trabalho deste conceito.

Quadro 2 - Conhecimentos Matemáticos para o Ensino de escala apresentada em gráficos

\begin{tabular}{|c|c|}
\hline $\begin{array}{l}\text { Tipos de Conhecimento } \\
\text { Docente }\end{array}$ & Habilidades Docentes \\
\hline \multirow{2}{*}{$\begin{array}{l}\text { Conhecimento Comum } \\
\text { de Escala }\end{array}$} & Conseguir localizar corretamente um valor na escala. \\
\hline & Construir corretamente a escala do gráfico. \\
\hline \multirow{4}{*}{$\begin{array}{l}\text { Conhecimento } \\
\text { Especializado de Escala }\end{array}$} & $\begin{array}{l}\text { Considerar as especificidades dos diferentes intervalos da } \\
\text { escala. }\end{array}$ \\
\hline & $\begin{array}{l}\text { Considerar a diferença de dificuldade em localizar um } \\
\text { valor implícito ou explícito na escala. }\end{array}$ \\
\hline & $\begin{array}{l}\text { Considerar que os diferentes conjuntos numéricos } \\
\text { influenciam no intervalo da escala. }\end{array}$ \\
\hline & $\begin{array}{l}\text { Considerar que a proporcionalidade é um atributo da } \\
\text { escala. }\end{array}$ \\
\hline $\begin{array}{l}\text { Conhecimento do } \\
\text { Horizonte de Escala }\end{array}$ & $\begin{array}{l}\text { Compreender como vai se complexificando a } \\
\text { compreensão do conceito de escala e os elementos que } \\
\text { influenciam nesta gradação. }\end{array}$ \\
\hline \multirow[t]{2}{*}{$\begin{array}{l}\text { Conhecimento de Escala } \\
\text { e do Aluno }\end{array}$} & $\begin{array}{l}\text { Compreender as dificuldades e facilidades dos alunos para } \\
\text { interpretar a escala. }\end{array}$ \\
\hline & $\begin{array}{l}\text { Compreender as dificuldades e facilidades dos alunos para } \\
\text { construir a escala. }\end{array}$ \\
\hline \multirow[t]{2}{*}{$\begin{array}{l}\text { Conhecimento de Escala } \\
\text { e Ensino }\end{array}$} & $\begin{array}{l}\text { Explorar o trabalho com as habilidades representar, } \\
\text { localizar, analisar, comparar e construir escalas em } \\
\text { diversas situações e com diferentes unidades escalares } \\
\text { durante as aulas. }\end{array}$ \\
\hline & $\begin{array}{l}\text { Variar as situações de ensino e uso do conceito de escala - } \\
\text { atividades de medida de comprimento, de reta numérica e } \\
\text { de mapas. }\end{array}$ \\
\hline $\begin{array}{l}\text { Conhecimento de Escala } \\
\text { e Currículo }\end{array}$ & $\begin{array}{l}\text { Conhecer o que os programas curriculares oficiais: BNCC } \\
\text { e PCN prescrevem para o trabalho com escala. }\end{array}$ \\
\hline
\end{tabular}




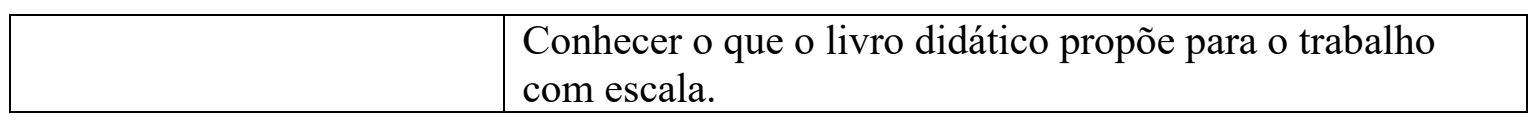

Fonte: as autoras

\section{Considerações Finais}

Este estudo partiu de uma necessidade atual inerente ao cenário educacional mundial para a compreensão de escalas apresentadas em gráficos, que é o desenvolvimento de um ensino que dê condições aos alunos de se constituírem cidadãos letrados estatisticamente. Temos uma mídia que faz uso constante das representações gráficas, muitas vezes apresentando informações manipuladas e na sala de aula encontramos alunos com muita dificuldade em compreender escala apresentada em gráficos e professores despreparados para o ensino. Além disso, se desejamos alunos capazes de compreender sobre o mundo físico e social, eles precisam saber sistematizar informações para tirar conclusões.

Nessa pesquisa tivemos como objetivo estabelecer relação entre os estudos anteriores que abordaram a escala apresentada em gráficos e a teoria Mathematical Knowledge for Teaching - MKT para definir os diferentes tipos de Conhecimentos Matemáticos para o Ensino de escala apresentada em gráficos estatísticos.

Para refletirmos sobre os saberes necessários para ensinar nos apoiamos em Ball et al. (2008), que nos apresentam seis Conhecimentos Matemáticos para o Ensino: Conhecimento Comum do Conteúdo, Conhecimento Especializado do Conteúdo, Conhecimento do Horizonte do Conteúdo, Conhecimento do Conteúdo e do Aluno, Conhecimento do Conteúdo e Ensino, Conhecimento do Conteúdo e Currículo.

Acreditamos que para que os professores sejam capazes de superar suas lacunas de formação em relação à escala apresentada em gráficos e possam levar seus alunos a também superar as dificuldades, não é suficiente o conhecimento matemático do conteúdo a ser ensinado. Consideramos, assim como Ball et al (2008), que existe uma multiplicidade de saberes que os docentes precisam mobilizar para o ensino de um conteúdo. Assim, defendemos que os professores precisam compreender e propor aos alunos um ensino considerando as habilidades levantadas por nós e apresentadas no Quadro 3, envolvendo os seis tipos de Conhecimentos matemáticos necessários para aprendizagem do conceito de escala nos anos iniciais.

Investigar o impacto deste modelo de processo de formação docente na aprendizagem de alunos precisa ser, ainda, realizado. 


\section{Referencias}

Ainley, J. (2000). Exploring the transparency of graphs and graphing. Proceeding 24nd Annual Meeting of the International Group for the Psychology of Mathematics Education, (1) South Africa.

Ainley, J.; Pratt, D.; Nardi, E. (2001). Normalising: children's activity to construct meanings for trend. Educational Studies in Mathematics, v. 45, n. 1-3, p. 131-146.

Araújo, L. C. (2007). Concepções e competências de um grupo de professores polivalentes relacionadas à leitura e interpretação de tabelas e gráficos. Dissertação (Mestrado Profissional) - Pontifícia Universidade Católica de São Paulo (PUC-SP), São Paulo.

Arteaga, P.; Batanero; Ruiz, B. (2008). Complejidad semiótica de gráficos estadísticos en La comparación de dos distribuciones por futuros profesores. Trabajo presentado en el Grupo de Probabilidad y Estadística. XII Simposio de la Sociedad Española de Educación Matemática. Badajoz.

Arteaga, P., Batanero, C. y Cañadas,G. (2011). Evaluación del conocimiento especializado de la estadística en futuros profesores en una tarea abierta. En M. Marín, G. Fernández, L. Blanco y M. Palarea (Eds.), Investigación en Educación Matemática XV. (pp. 267-275). Ciudad Real: SEIEM.

Ball, D.; Bass, H. (2003).Toward a Practice-Based Theory of Mathematical Knowledge for Teaching. In: DAVIS, B.; SIMMT, E. (Ed.). Proceedings of the 2002 Annual Meeting of the Canadian Mathematics Education Study Group, p. 3-14

Ball, D. L.; Thames, M. H.; Phelps, G. (2008). Content Knowledge for Teaching: what makes it special? In: Journal of teacher educacion, v. 59, n. 5, p. 389-407.

Brasil. (1997). Secretaria de Educação Fundamental. Parâmetros Curriculares Nacionais: Matemática, Ensino de $1^{a}$ à $4^{a}$ série. Brasília, MEC/ SEF.

Brasil. (2015). Guia de livros didáticos: PNLD 2016: Alfabetização Matemática e Matemática: ensino fundamental anos iniciais. Brasília: Ministério da Educação, Secretária de Educação Básica.

Brasil. (2018). Ministério da Educação. Base Nacional Comum Curricular. Brasília: MEC.

Cavalcanti, M. (2010). Como adultos e crianças compreendem a escala representada em gráficos. Dissertação (Mestrado em Educação Matemática e Tecnológica) - UFPE, Recife.

Cavalcanti, M.; Natrielli, K. R.; Guimarães, G. (2010). Gráficos na mídia impressa. Boletim de Educação Matemática - Bolema, v. 23, p. 733 -752.

Cavalcanti, M ; Guimarães, G. L. (2016). Compreensão de Adultos e Crianças sobre Escala Representada em Gráficos. Perspectivas da Educação Matemática, v. 9, p. 849-868.

Díaz, D.; Batanero, C.; Arteaga, P.; Serrano, M. (2015). Análisis de gráficos estadísticos em libros de texto de educación primaria española. Revista Iberoamericana de Educação Matemática-UNIÓN. n. 44, p. 90-112.

Evangelista, M. B.; Guimarães, G. (2013). Análise de atividade de livros didáticos de matemática do $4^{\circ}$ e $5^{\circ}$ ano que exploram o conceito de escala. VII Congreso Iberomericano de Educación Matemática - CIBEM. Montevideo.

Evangelista, M. B. (2014). Aprendendo a representar escalas em gráficos: um estudo de intervenção. 2014. Dissertação (Mestrado em Educação Matemática e Tecnológica) - 
UFPE. Recife.

Friel, S.; Curcio, F.; Bright, G. (2001). Making Sense of Graphs: critical factors influencing comprehension and instructional implications. Journal for Research in Mathematics Education, v. 32, n. 2, p. 124-158.

Gal, I. (2002). Adults statistical literacy: meanings, components, responsibilities. International Statistical Review, v. 70, n. 1, p. 1-25.

Guimarães, G. L. (2016). Aprendizagens na vida e na escola: estatística nos anos iniciais. Anais do Simpósio Internacional de Pesquisa em Educação Matemática - SIPEM, São Paulo.

Guimarães, G. L. (2010). Formação de professores: trabalhando com gráficos e tabelas na educação infantil. Revista Iberoamericana de Educação Matemática - UNIÓN, n. 21, p. 87-101.

Guimarães, G. L.; Gitirana, V.; Roazzi, A. (2001). Interpretando e construindo gráficos. Anais da $24^{a}$ Reunião Anual da ANPED, Caxambu.

Guimarães, G. L.; Gitirana, V.; Cavalcanti, M.; Marques, M. (2007). Livros didáticos de matemática nos anos iniciais: análise das atividades sobre gráficos e tabelas. Anais do $I X$ Encontro Nacional de Educação Matemática. Belo Horizonte.

Inaf (2016). Indicador nacional de alfabetismo funcional. São Paulo: Ação Educativa.

Lemos, M. P.; Gitirana, V. (2007). Interpretação de gráficos de barras: analise a priori enquanto recurso na formação de professores. Anais do Encontro Nacional de Educação Matemática. Belo Horizonte.

Lima; Magina, S. (2004). A leitura de gráficos com crianças da $4^{\mathrm{a}}$ série do Ensino Fundamental. Anais do Encontro Nacional de Educação Matemática.

Lima, A. P.; Silva, E; Rodrigues, J.; Feitoza, L. (2006). Interpretação e construção de gráficos de barras. Pôster apresentado na disciplina Metodologia do Ensino de Matemática I do Curso de Pedagogia.

Lima, I. B. (2010). Investigando o desempenho de jovens e adultos na construção e interpretação de gráficos. Dissertação (Mestrado em Educação Matemática e Tecnológica) - UFPE. Recife.

Monteiro, C.; Selva, A. (2001). Investigando a Atividade de Interpretação de Gráficos entre Professores do Ensino Fundamental. Anais da $24^{a}$ Reunião Anual da Associação Nacional de Pós-Graduação e Pesquisa em Educação - Anped. Caxambu/MG.

Pagan, A.; Leite, A. P.; Magina, S.; Cazorla, I. (2008). A leitura e interpretação de gráficos e tabelas no Ensino Fundamental e Médio. Anais do $2^{\circ}$ Simpósio Internacional de Pesquisa em Educação Matemática (SIPEMAT). Recife.

Patrocínio, A.; Guimarães, G. (2007). Relação entre representações gráficas e escolarização. Anais do Encontro Nacional de Educação Matemática, Belo Horizonte.

Rossine, R. (2006). Saberes docentes sobre o tema Função: uma investigação das praxeologias. Tese (Doutorado em Educação Matemática) - Pontifícia Universidade Católica. São Paulo.

Selva, A. (2003). Um experimento de ensino sobre a resolução de problemas de estrutura aditiva a partir de gráficos de barras. Anais da $26^{a}$ Reunião Anual da ANPED. Caxambu, MG.

Shaughnessy, J.; Garfield, J.; Greer, B. (1996). Data Handling. Bishop, A. et al. (eds.), International handbook on mathematics education. Netherlands: Kluwer, p. 205-237. 
Silva, F.; Amaral, F.; Albuquerque, J.; Oliveira, L. (2006). Gráficos: importante ou determinante, eis a avaliação do professor. Pôster apresentado na disciplina Metodologia do Ensino de Matemática I, do Curso de Pedagogia.

Tierney, C.; Nemirovsky, R. (1991). Children's spontaneous representations of changing situations. Hands on!, v. 4, n. 2, p. 7-10.

Tierney, C.; Weinberg, A.; Nemirovsky, R. (1992). Telling Stories Plant Growth: Fourth Grade Students Interpret Graphs. XVI Proceedings of de Annual Meeting of the International Group for the Psychology of Mathematics Education (PME, N. H., USA).

Vanin. (2009). Gráficos, Proporções e Variações Proporcionais. Disponível em: https://edisciplinas.usp.br/pluginfile.php $/ 2565417 / \mathrm{mod}$ _resource/content $/ 3 /$ TExto $\% 20 \mathrm{co}$ mplementar\%201.pdf. Acesso em: 18 maio 2017. 